\title{
A influência do Delirium no tempo de ventilação mecânica em pacientes críticos: uma revisão sistemática
}

\author{
The influence of Delirium in the time of mechanical ventilation in critical \\ patients: a systematic review
}

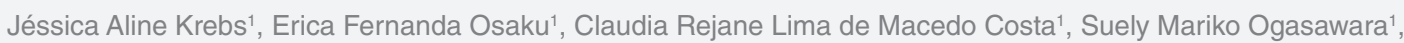
Jaqueline Barreto da Costa ${ }^{1}$, Sheila Taba ${ }^{1}$, Amaury Cezar Jorge ${ }^{1}$, Pericles Almeida Delfino Duarte ${ }^{1}$

Universidade Estadual do Oeste do Paraná (Unioeste) - Cascavel (PR), Brasil.

DOI: https://dx.doi.org/10.7322/abcshs.v43i1.1028

\section{RESUMO}

O delirium consiste em um estado confusional agudo e de curso flutuante representando uma manifestação da disfunção cerebral que pode estar associado com diferentes manifestações clínicas. Os pacientes com delirium têm sido estudados, pois tem apresentado maior tempo de ventilação mecânica e de internação em unidade de terapia intensiva. O objetivo do estudo foi realizar uma revisão de literatura acerca da influência do delirium no tempo de ventilação mecânica, sedação e na mortalidade de pacientes internados em unidade de terapia intensiva. Foi realizada uma busca por estudos nas bases de dados PubMed e Embase, com os descritores delirium, intensive care unit and mechanical ventilation. Nos resultados foram incluídos oito artigos, um recebeu graduação $A$ e sete receberam graduação B na escala de Oxford. Os principais resultados foram: Os pacientes com delirium apresentaram maior gravidade, maior tempo de ventilação mecânica, maior tempo de sedação e maior mortalidade. Conclui-se que o delirium parece estar associado ao maior tempo da ventilação mecânica, maior dosagem de sedação e de mortalidade nestes pacientes.

Palavras-chave: Delírio; unidade de terapia intensiva; respiração artificial.

\begin{abstract}
Delirium consists of an acute, fluctuating, confusional state, representing a manifestation of cerebral dysfunction that can occur with different clinical manifestations. Patients with delirium have been studied because they have had longer time of mechanical ventilation and hospitalization in the intensive care unit. The objective of the study was to perform a literature review about the influence of delirium on the time of mechanical ventilation, sedation and on the mortality of patients admitted in intensive care units. A search for studies with the keywords delirium, intensive care unit and mechanical ventilation was performed in PubMed and Embase databases. As result eight articles were included, one received level $A$ and seven received level $B$ on the Oxford scale. The main results were: Patients with delirium presented greater severity, longer time of mechanical ventilation, longer sedation time and higher mortality. It is concluded that delirium appears to be associated with longer mechanical ventilation, greater sedation and mortality rates in these patients.
\end{abstract}

Keywords: Delirium; intensive care unit; respiration, artificial.

Recebido em: 08/09/2017

Revisado em: 26/11/2017

Aprovado em: 30/11/2017

Autora para correspondência: Jéssica Aline Krebs - Universidade Estadual do Oeste do Paraná - Rua Universitária, 1619 - Universitário - CEP: 85819 -110 Cascavel (PR), Brasil - E-mail: jeekrebs@gmail.com

Conflito de interesses: nada a declarar. 


\section{INTRODUÇÃO}

O delirium na unidade de terapia intensiva (UTI) vem sendo estudado cada vez mais devido a sua complexidade e por permanecer ainda sub-diagnosticado ${ }^{1}$. Na maioria das UTIs existem fatores que levam a insônia, isolamento e uma privação do ambiente externo e sua luminosidade ao paciente ${ }^{2}$. As taxas gerais de incidência de delírium na UTI foram relatadas ${ }^{3,4}$ entre $11 \%$ a $87 \%$.

Conforme Diagnostic and Statistical Manual of Mental Disorders (DSM IV) o delirium é definido como uma alteração do consciente, reduzindo a aptidão de evidenciar, preservar ou alterar a atenção. É caracterizado como uma modificação na parte cognitiva ou na evolução de transtornos da percepção que não possam ser associados a alterações já existentes. De acordo com o DSM-IV, todos esses critérios devem estar presentes para se diagnosticar delirium ${ }^{5}$.

A nova versão do DSM-V determina o subtipo de delirium, que pode ser por intoxicação por substância, abstinência de substância ou induzido por medicamentos. Pode se especificar que é agudo (duração de poucas horas a dias) e persistente (duração de semanas a meses), e ainda dividir em hiperativo (nível hiperativo de atividade psicomotora que pode ser acompanhado de oscilação de humor, agitação e/ou recusa a cooperar com os cuidados médicos), hipoativo (nível hipoativo de atividade psicomotora que pode estar acompanhada de lentidão e letargia que se aproxima do estupor) e nível misto (nível normal de atividade psicomotora mesmo com perturbação da atenção e da percepção).

O delirium é uma causa não pulmonar que continua a ser estudada em pacientes criticamente enfermos por aumentar o tempo de ventilação mecânica (VM) com incidencia ${ }^{4}$ entre 60 a $80 \%$. $\mathrm{O}$ delirium tem sido relacionado a resultados piores em pacientes que necessitam de cuidados intensivos, aumentando a duração do tempo de permanência no hospital e mortalidade $e^{4,6,7}$.

Algumas intervenções tem demostrado reduzir o risco de delirium e o tempo de VM entre os pacientes na UTI, como a retirada diária da sedação, a realização do teste de respiração espontânea, a avaliação do delirium três vezes ao dia, a mobilização precoce $e^{8-11}$ e o uso de ventilação não invasiva ${ }^{12}$. Sendo assim, o objetivo desse trabalho foi realizar um levantamento da produção cientifica acerca da influência do delirium no tempo de VM, no tempo de sedação e na mortalidade de pacientes internados em UTI.

\section{MÉTODOS}

O presente estudo foi desenvolvido por meio de uma revisão de literatura, conduzido através de informações obtidas nas bases de dados PubMed e Embase.

Os termos utilizados, como palavras-chave, foram: Delirium, intensive care unit (ICU) e mechanical ventilation. A revisão buscou em artigos científicos as palavras chaves e suas similaridades nas bases de dados citadas acima, conforme os níveis de evidência.

Os estudos foram analisados e classificados conforme a recomendação de "Oxford Centre for Evidence-Based Medicine": que distribui os estudos de um a cinco e formam graus de recomendação, sendo A (artigos de classificação nível 1), B (classificação 2 e 3), C (classificação 4) e D (classificação 5)(Oxford) ${ }^{13}$.

Como critérios de inclusão, as populações dos estudos selecionados foram compostas por pacientes adultos de ambos os sexos, apresentando comparação entre tempo de VM de pacientes com e sem delirium, que receberam ventilação mecânica associada à sedação por curto ou longo tempo. Não houve limitação do período de tempo para inclusão dos artigos pela pequena quantidade de estudos potencialmente relevantes.

Foram excluídos estudos que continham apenas os resumos nas bases de dados, artigos que não tinham grupo de pacientes sem delirium, relatos de casos e estudos que apresentassem pacientes com diagnóstico prévio de transtorno psiquiátrico, etilistas e dependentes químicos.

\section{RESULTADOS}

A pesquisa realizada encontrou 478 resumos potencialmente relevantes, sendo que 315 artigos foram selecionados para a análise detalhada. Após a leitura dos textos, oito artigos preencheram os critérios e foram incluídos na revisão sistemática (Figura 1).

A ferramenta mais usada para o diagnóstico de delirium foi o CAM-ICU (Confusion Assessment Method for Intensive Care Unit), utilizada em seis estudos ${ }^{7,14-18}$, sendo que em um foi utilizado o CAM-ICU e ICDSC (Intensive Care Delirium Screening Checklist) aleatoriamente ${ }^{19}$, e em outro estudo ${ }^{20}$ foi aplicado o ICDSC isoladamente, de acordo com o Quadro 1.

Entre os artigos selecionados, observou-se que as amostras são compostas por pacientes mais idosos, sendo todos com média de idade maior que 50 anos em ambos os grupos, e sua grande maioria do sexo masculino (Quadro 1).

O desfecho de mortalidade não apresentou diferença estatística entre os grupos em cinco estudos ${ }^{14,15,19,20}$, divergindo com quatro

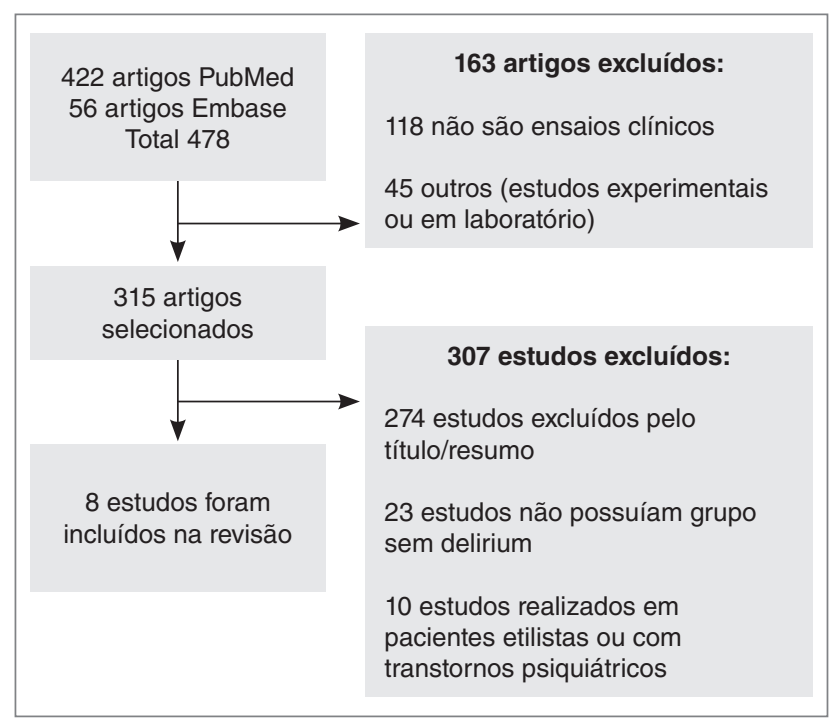

Figura 1: Diagrama de seleção dos artigos 
estudos que mostraram aumento da mortalidade no grupo delirium $(\mathrm{GD})^{7,15,17,18}$. Os pacientes do GD permaneceram mais dias em UTI em relação ao grupo não delirium (GND) e apenas em um estudo não houve diferença entre os grupos ${ }^{17}$.

A gravidade da doença foi avaliada pelo escore APACHE II em cinco estudos, em um estudo APACHE II e SOFA, em um artigo foi utilizado APACHE III e SOFA e um artigo usaram o SOFA isoladamente. Todos os artigos relataram que os pacientes com delirium apresentaram maior gravidade. Quanto ao uso de opióides a maioria dos estudos mostrou que os pacientes do $\mathrm{GD}^{7,16,17,20}$ utilizaram maior dosagem e quantidade de sedação (Quadro 2). As drogas mais utilizadas para sedação foram Midazolam e Lorazepan, com dosagens maiores no GD e os opiáceos utilizados foram Fentanil em quatro estudos ${ }^{7,16,17,20}$ e Propofol em dois estu$\operatorname{dos}^{7,16}$. Os demais estudos não relataram a dosagem das drogas, apenas a quantidade de dias de sedação.

O tempo de VM foi maior no GD na maioria dos estudos ${ }^{14-17,20}$, sendo que em 3 estudos também foi relatado dados sobre a extubação $0^{13,14,19}$, apresentando maior probabilidade de extubação no GND (Quadro 2).

Quadro 1: Características dos artigos selecionados

\begin{tabular}{|c|c|c|c|c|c|}
\hline Autor/Ano & $\begin{array}{l}\text { Oxford/ } \\
\text { Tipo de estudo }\end{array}$ & Intrumento utilizado & Amostra & $\begin{array}{c}\text { Idade } \\
\text { (média/DP ou IQR) }\end{array}$ & Sexo Masculino \\
\hline $\begin{array}{l}\text { Jeon et al. } \\
2016^{14}\end{array}$ & $\begin{array}{l}\text { B/Prospectivo, } \\
\text { observacional }\end{array}$ & CAM - ICU & $\begin{array}{c}160 \text { (GD) } \\
233 \text { (GND) }\end{array}$ & $\begin{array}{c}\text { 70(61-75) (GD) } \\
61(47-71)(G N D)\end{array}$ & $\begin{array}{c}109 \text { (GD) } \\
163 \text { (GND) }\end{array}$ \\
\hline $\begin{array}{l}\text { Lat et al. } \\
2009^{16}\end{array}$ & $\begin{array}{l}\text { B/Prospectivo, } \\
\text { observacional }\end{array}$ & CAM - ICU & $\begin{array}{c}84 \text { (GD) } \\
50 \text { (GND) }\end{array}$ & $\begin{array}{c}53,5(32-70)(G D) \\
50,5(34-73) \text { (GND) }\end{array}$ & $\begin{array}{c}55 \text { (GD) } \\
32 \text { (GND) }\end{array}$ \\
\hline $\begin{array}{l}\text { Lin et al. } \\
2008^{15}\end{array}$ & $\begin{array}{l}\text { B/Prospectivo, } \\
\text { observacional }\end{array}$ & CAM - ICU & $\begin{array}{c}31 \text { (GD) } \\
112 \text { (GND) }\end{array}$ & $\begin{array}{c}76(64-85)(G D) \\
76(66-84) \text { (GND) }\end{array}$ & $\begin{array}{c}14 \text { (GD) } \\
72 \text { (GND) }\end{array}$ \\
\hline $\begin{array}{l}\text { Mehta et al. } \\
2015^{20}\end{array}$ & $\begin{array}{l}\mathrm{A} / \text { Estudo } \\
\text { randomizado }\end{array}$ & ICDSC & $\begin{array}{c}226 \text { (GD) } \\
163 \text { (GND) }\end{array}$ & $\begin{array}{c}57(46-68)(G D) \\
60(50-71)(G N D)\end{array}$ & $\begin{array}{l}138 \text { (GD) } \\
76 \text { (GND) }\end{array}$ \\
\hline $\begin{array}{l}\text { Micek et al. } \\
2005^{17}\end{array}$ & $\begin{array}{l}\text { B/Prospectivo, } \\
\text { observacional }\end{array}$ & CAM - ICU & $\begin{array}{c}44 \text { (GD) } \\
22 \text { (GND) }\end{array}$ & $\begin{array}{c}59(47-73)(G D) \\
62(48-73)(G N D)\end{array}$ & $\begin{array}{c}20 \text { (GD) } \\
10 \text { (GND) }\end{array}$ \\
\hline $\begin{array}{l}\text { Spronk et al. } \\
2009^{19}\end{array}$ & $\begin{array}{l}\text { B/Prospectivo, } \\
\text { observacional }\end{array}$ & CAM - ICU ou ICDSC & $\begin{array}{c}23 \text { (GD) } \\
23 \text { (GND) }\end{array}$ & $\begin{array}{c}\text { 74(71-81) (GD) } \\
71(58-80) \text { (GND) }\end{array}$ & $\begin{array}{c}16 \text { (GD) } \\
14 \text { (GND) }\end{array}$ \\
\hline $\begin{array}{l}\text { Tsuruta et al. } \\
2010^{18}\end{array}$ & $\begin{array}{l}\text { B/Prospectivo, } \\
\text { observacional }\end{array}$ & CAM - ICU & $\begin{array}{c}21(\mathrm{GD}) \\
82 \text { (GND) }\end{array}$ & $\begin{array}{c}70 \pm 17 \text { (GD) } \\
64 \pm 19 \text { (GND) }\end{array}$ & $\begin{array}{c}62(\mathrm{GD}) \\
62 \text { (GND) }\end{array}$ \\
\hline $\begin{array}{l}\text { Ely et al. } \\
2004^{7}\end{array}$ & $\begin{array}{l}\text { B/Prospectivo, } \\
\text { observacional }\end{array}$ & CAM - ICU & $\begin{array}{l}183 \text { (GD) } \\
41 \text { (GND) }\end{array}$ & $\begin{array}{l}56 \pm 17 \text { (GD) } \\
54 \pm 9 \text { (GND) }\end{array}$ & $\begin{array}{c}95(\mathrm{GD}) \\
18 \text { (GND) }\end{array}$ \\
\hline
\end{tabular}

GD: Grupo delirium; GND: Grupo não delirium; CAM - ICU: Confusion Assessment Method for Intensive Care Unit; ICDSC: Intensive Care Delirium Screening Checklist; DP: Desvio Padrão; IQR: Amplitude Interquantil.

Quadro 2: Caracteristicas dos pacientes

\begin{tabular}{|c|c|c|c|c|c|c|c|c|c|}
\hline $\begin{array}{l}\text { Autor/ } \\
\text { Ano }\end{array}$ & Dias UTI & APACHE II & SOFA & $\begin{array}{l}\text { Duração da } \\
\text { VM (dias) }\end{array}$ & Extubação & Mortalidade & $\begin{array}{l}\text { Benzodia- } \\
\text { zepínicos } \\
\text { (dose) }\end{array}$ & \multicolumn{2}{|c|}{ Opióides (dose) } \\
\hline $\begin{array}{l}\text { Jeon et al. } \\
2015^{14}\end{array}$ & $\begin{array}{l}10(G D) \\
6 \text { (GND) } \\
p<0,001\end{array}$ & - & $\begin{array}{c}9 \text { (GD) } \\
6 \text { (GND) } \\
p<0,01\end{array}$ & $\begin{array}{l}3,5 \text { (GD) } \\
3 \text { (GND) } \\
p=0,003\end{array}$ & $\begin{array}{c}39 \text { (GD) } \\
32 \text { (GND) } \\
p=0,005\end{array}$ & - & 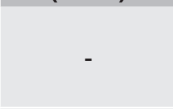 & \multicolumn{2}{|l|}{ 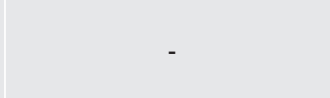 } \\
\hline $\begin{array}{l}\text { Lat et al. } \\
2009^{16}\end{array}$ & $\begin{array}{c}10(\mathrm{GD}) \\
5(\mathrm{GND}) \\
\mathrm{p}<0.01\end{array}$ & $\begin{array}{c}20 \text { (GD) } \\
17,7 \text { (GND) }\end{array}$ & - & $\begin{array}{c}7,5 \text { (GD) } \\
3 \text { (GND) } \\
p \leq 0,01\end{array}$ & - & - & $\begin{array}{l}\text { Lorazepam } \\
\text { GD 3,0 mg } \\
\text { GND 2,0 mg }\end{array}$ & $\begin{array}{c}\text { Propofol } \\
\text { GD, GND } \\
0.5 \mathrm{mg}\end{array}$ & $\begin{array}{c}\text { Fentanil } \\
\text { GD } 1,1 \mathrm{mg} \\
\text { GND } 0,9 \mathrm{mg}\end{array}$ \\
\hline $\begin{array}{l}\text { Lin et al. } \\
2008^{15}\end{array}$ & $\begin{array}{l}13 \text { (GD) } \\
8 \text { (GND) }\end{array}$ & $\begin{array}{c}\text { APACHE III } \\
61 \text { (GD) } \\
64 \text { (GND) }\end{array}$ & $\begin{array}{c}8 \text { (GD) } \\
6 \text { (GND) }\end{array}$ & $\begin{array}{l}16 \text { (GD) } \\
6 \text { (GND) } \\
p=0,003\end{array}$ & $\begin{array}{c}6(G D) \\
22 \text { (GND) } \\
p=0,001\end{array}$ & $\begin{array}{c}21 \text { (GD) } \\
38 \text { (GND) }\end{array}$ & - & \multicolumn{2}{|c|}{ - } \\
\hline $\begin{array}{l}\text { Mehta et al. } \\
2015^{20}\end{array}$ & $\begin{array}{c}12 \text { (GD) } \\
8 \text { (GND) } \\
p=0.0001\end{array}$ & $\begin{array}{c}23 \text { (GD) } \\
24 \text { (GND) } \\
p=0,40\end{array}$ & - & $\begin{array}{l}13 \text { (GD) } \\
7 \text { (GND) } \\
p \leq 0,0001\end{array}$ & $\begin{array}{l}21(G D) \\
2 \text { (GND) } \\
\mathrm{p}=0,0003\end{array}$ & $\begin{array}{c}40 \text { (GD) } \\
37 \text { (GND) }\end{array}$ & $\begin{array}{c}\text { Midazolam } \\
104 \mathrm{mg} \text { (GD) } \\
57 \mathrm{mg}(\mathrm{GND}) \\
\mathrm{p}<0,0001\end{array}$ & \multicolumn{2}{|c|}{$\begin{array}{c}\text { Fentanil } \\
\text { GD } 1,497 \mathrm{mg} \\
\text { GND } 1,150 \mathrm{mg} \\
p<0,0001\end{array}$} \\
\hline $\begin{array}{l}\text { Micek et al. } \\
2005^{17}\end{array}$ & $\begin{array}{c}9,5 \text { (GD) } \\
9,5 \text { (GND) }\end{array}$ & $\begin{array}{c}20 \text { (GD) } \\
19 \text { (GND) }\end{array}$ & - & $\begin{array}{c}6,7 \text { (GD) } \\
3,8 \text { (GND) } \\
p=0,004\end{array}$ & - & $\begin{array}{c}14 \text { (GD) } \\
8 \text { (GND) } \\
p=0.5\end{array}$ & $\begin{array}{l}\text { Midazolam } \\
\text { GD } 247 \mathrm{mg} \\
\text { GND } 200 \mathrm{mg}\end{array}$ & \multicolumn{2}{|c|}{$\begin{array}{c}\text { Fentanil } \\
\text { GD } 11,885 \mathrm{mg} \\
\text { GND } 7,600 \mathrm{mg}\end{array}$} \\
\hline $\begin{array}{l}\text { Spronk et al. } \\
2009^{19}\end{array}$ & $\begin{array}{c}9 \text { (GD) } \\
5 \text { (GND) }\end{array}$ & $\begin{array}{c}18 \text { (GD) } \\
16 \text { (GND) }\end{array}$ & - & $\begin{array}{c}6(G D) \\
4 \text { (GND) } \\
p=0,013\end{array}$ & - & $\begin{array}{c}6 \text { (GD) } \\
5 \text { (GND) }\end{array}$ & 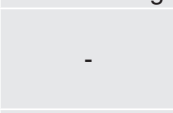 & \multicolumn{2}{|c|}{ - } \\
\hline $\begin{array}{l}\text { Tsuruta et al. } \\
2010^{18}\end{array}$ & $\begin{array}{l}12 \text { (GD) } \\
5 \text { (GND) } \\
p=0,001\end{array}$ & $\begin{array}{c}17 \text { (GD) } \\
11(\text { GND) } \\
p=0,001\end{array}$ & - & - & - & $\begin{array}{c}2 \text { (GD) } \\
0 \text { (GND) } \\
p=0,05\end{array}$ & - & \multicolumn{2}{|c|}{ - } \\
\hline $\begin{array}{l}\text { Ely et al. } \\
2004^{7}\end{array}$ & $\begin{array}{c}7 \text { (GD) } \\
5 \text { (GND) } \\
p=0,09\end{array}$ & $\begin{array}{c}25,6 \text { (GD) } \\
23,3 \text { (GND) }\end{array}$ & $\begin{array}{c}9,6 \text { (GD) } \\
9,5 \text { (GND) }\end{array}$ & -- & -- & $\begin{array}{l}63(\mathrm{GD}) \\
6 \text { (GND) }\end{array}$ & $\begin{array}{l}\text { Lorazepan } \\
\text { GD } 49,2 \mathrm{mg} \\
\text { GND } 9,0 \mathrm{mg}\end{array}$ & $\begin{array}{c}\text { Propofol } \\
\text { GD } 591 \mu \mathrm{g} \\
\text { GND } 362 \mu \mathrm{g}\end{array}$ & $\begin{array}{c}\text { Fentanil } \\
\text { GD } 8,7 \mathrm{mg} \\
\text { GND } 3,1 \mathrm{mg}\end{array}$ \\
\hline
\end{tabular}

GD: Grupo delirium; GND: Grupo não delirium; VM: ventilação mecânica; UTI: Unidade de Terapia Intensiva; APACHE II: Acute Physiology And Chronic Health Evaluation II; SOFA: Sepsis-related Organ Failure Assessment. 


\section{DISCUSSÃo}

\section{Tempo de ventilação mecânica e delirium}

O tempo de VM é uma variável importante no prognóstico do paciente ${ }^{21}$ sendo que o tempo prolongado está associado ao aumento da morbidade e mortalidade, conforme os estudos analisados.

Em três estudos ${ }^{14,15,20}$ foi relatado a falência de extubação, não apresentando diferença na falha de extubação entre os grupos GD e GND. Porém, após o sucesso de um teste de respiração espontânea (TRE), os pacientes com delirium apresentavam menor probabilidade de serem extubados em comparação com pacientes sem delirium ${ }^{20}$ fazendo com que os pacientes com delirium permanecessem mais tempo em VM.

O delirium tem recebido pouca atenção no processo de desmame, porém estudos têm mostrado relação entre o tempo de VM e a presença de delirium nesse período importante de transição do paciente para respiração espontânea, sendo extremamente crucial a preservação da função cerebral ${ }^{22}$.

Um estudo realizado na Coreia ${ }^{13}$ avaliou o desmame da VM em pacientes com delirium. Entre os achados do estudo, a taxa de extubação no primeiro teste de respiração espontânea (TRE) foi maior entre os pacientes com CAM-ICU (-) do que entre aqueles com CAM-ICU (+), e a falha de extubação e reintubação também foram maiores em pacientes com CAM-ICU $(+)$ do que naqueles com CAM-ICU (-) concordando com resultados de outros estudos que avaliaram as taxas de extubação, mostrando que esses pacientes que falham na extubação voltam para VM, aumentando o tempo com suporte ventilatório nos pacientes com delirium ${ }^{15,19}$.

O tempo de VM foi significativamente maior na maioria dos estudos em pacientes com delirium ${ }^{14-17,20}$ concordando com outros estudos que mostraram que o delirium esta associado ao maior tempo de $\mathrm{VM}^{5,7}$. A incidência de delirium nos pacientes em $\mathrm{VM}^{23}$ é em torno de $50 \%$.

Segundo Tsuruta et al. ${ }^{18}$ o delirium pode se manifestar em até $80 \%$ devido a gravidade da doença, aumento do tempo de sedação e consequentemente maior tempo de internamento hospitalar. Ainda de acordo com Tsuruta et al. ${ }^{18}$ a prevalência de delirium em pacientes que não utilizaram da VM é de $20 \%$, justificando as baixas taxas de delirium em UTIs com menos pacientes em VM.

Observou-se nos artigos que os pacientes com delirium tiveram resultados piores do que pacientes sem delirium, incluindo maior tempo de estadia na UTI ${ }^{14,16,18-20}$ sendo que um estudo comprovou que a VM e o tempo de internamento na UTI são fatores independentes para o desenvolvimento de delirium ${ }^{14}$.

Os estudiosos Jeon et al. ${ }^{14}$ e Tsuruta et al..$^{18}$ defendem a ideia de que devido à gravidade da doença desses pacientes com delirium há a necessidade de maior tempo de VM com o objetivo de tratar a causa da insuficiência respiratória que levou esses pacientes a serem intubados. Deve-se ter em mente que a VM é de suma importância quando o paciente não possui mais condições de manter uma boa oxigenação ou proteger vias aéreas, porém o tempo prolongado utilizando o dispositivo ventilatório pode desenvolver o delirium ${ }^{14,18}$.

\section{Sedação e delirium}

Os benzodiazepínicos são frequentemente utilizados no ambiente de cuidados intensivos. A exposição do paciente aos benzodiazepínicos tem sido implicada no desenvolvimento e na duração do delirium. Durante a última década, as evidências são que o uso de benzodiazepínicos para sedação de pacientes internados em UTI, especificamente Midazolam e Lorazepam, estão associados a resultados piores em relação a tempo de VM, dias de UTI e aumento de delirium do que a sedação com os não benzodiazepínicos, especialmente Propofol e Dexmedetomidina ${ }^{24,25}$.

Nesta revisão, apenas um estudo avaliou o uso de Lorazepam em relação ao delirium, sendo que os pacientes que o utilizaram, eram significativamente mais delirantes ${ }^{16}$ concordando com Pandharipande et $a l .{ }^{6}$ que encontrou resultados semelhantes, mostrando um aumento do delirium em pacientes que usaram Lorazepam $^{26}$. Existe uma concordância entre alguns estudos ${ }^{27,28}$ que mostram que as benzodiazepinas são um fator de risco independente para o desenvolvimento de delirium durante a doença crítica.

Estudos randomizados e controlados evidenciaram que os pacientes com interrupção diária de sedação receberam menor quantidade de sedação e tiveram menos dias em VM, menor tempo de internação na UTI e menos dias de delirium ${ }^{29,30}$. Segundo Vasilevskis et al. ${ }^{30}$ uma minoria de pacientes de UTI tem indicação de sedação profunda e contínua, como nos tratamento da hipertensão intracraniana e insuficiência respiratória grave.

Houve concordância entre os resultados de dois estudos ${ }^{17,20}$ em relação ao uso do Midazolam, onde um número significativo de pacientes com delirium recebeu maior dosagem e tempo de sedação contínua em comparação com pacientes sem delirium. Resultados diferentes foram encontrados no estudo de Leite et al. ${ }^{31}$ que mostrou que as altas doses de Midazolam não influenciaram na ocorrência de delirium.

Dois estudos relevantes foram publicados comparando a incidência de delirium em pacientes na UTI que receberam propofol versus benzodiazepinas para sedação e não mostrando diferença significativa entre eles em relação ao delirium ${ }^{32,33}$.

Uma meta-análise ${ }^{34}$ realizada para observar os desfechos na comparação de propofol e midazolam na mortalidade não apresentou diferença significativa e mostrou apenas redução na duração da ventilação mecânica com o uso de propofol.

\section{Mortalidade e delirium}

O desenvolvimento de delirium foi associado com desfechos clínicos graves, porém a associação entre delirium e mortalidade não é clara. Três estudos ${ }^{14,15,18}$ encontraram um aumento da mortalidade em pacientes delirantes, variando de 18 a 32\%, concordando com a literatura que mostra que a mortalidade gira em torno de $30 \%$ nesses pacientes ${ }^{7}$.

A meta-analise de Salluh et al. ${ }^{1}$ avaliou em 28 estudos a mortalidade e a taxa de risco de morte, sendo que em pacientes com delirium esses indicativos foram significativamente maiores. Os autores ainda relatam maior gravidade dos pacientes delirantes 
avaliados através do APACHE II, justificando a maior taxa de mortalidade nestes pacientes. No entanto, os autores declaram que não é claro se estes resultados estão relacionados a complicações diretas do delirium.

O delirium tem causado grandes alterações na qualidade de vida e nos desfechos clínicos nos pacientes que sobrevivem à UTI. Os pacientes com delirium hipoativo têm seu risco de mortalidade triplicado em seis meses ${ }^{7}$.

Seis em cada dez pacientes que sobrevivem à doença crítica apresentam de meses a anos de comprometimento cognitivo após alta da UTI. Esse paciente tem apresentado demência adquirida e deficiência cognitiva em longo prazo e isso tem levado a reduzir significativamente a qualidade de vida ${ }^{35}$.

\section{CONSIDERAÇÕES FINAIS}

Pode-se observar que o delirium está presente em uma ampla amostra de paciente em estado crítico, sendo a maioria do sexo masculino que utilizaram maior dosagem de sedação e consequentemente permaneceram mais tempo em ventilação mecânica. O delirium somado a todos esses fatores pode aumentar a mortalidade nessa população.

\section{REFERÊNCIAS}

1. Salluh JIF, Wang H, Schneider EB, Nagaraja N, Yenokyan G, Damluji $\mathrm{A}$, et al. Outcome of delirium in critically ill patients: systematic review and meta-analysis. BMJ. 2015;350:h2538. https://dx.doi.org/10.1136/bmj.h2538

2. Inouye SK, Bogardus Jr ST, Charpentier PA, Leo-Summers L, Acampora D, Holford TR, et al. A multicomponent intervention to prevent delirium in hospitalized older patients. N Engl J Med. 1999: 340(9):669-76. https://dx.doi.org/10.1056/NEJM199903043400901

3. Aldemir M, Özen S, Kara IH, Sir A, Baç B. Predisposing factors for delirium in the surgical intensive care unit. Crit Care. 2001;5(5):265-70. https://dx.doi.org/10.1186/cc1044

4. Ely EW, Inouye SK, Bernard GR, Gordon S, Francis J, May L, et al. Delirium in mechanically ventilated patients: validity and reliability of the confusion assessment method for the intensive care unit (CAM-ICU). JAMA. 2001;286(21):2703-10.

https://dx.doi.org/10.1001/jama.286.21.2703

5. Associação Psiquiátrica Americana. Manual diagnóstico e estatístico de transtornos mentais: DSM-4. $4^{\mathrm{a}}$ ed. Porto Alegre: Artes Médicas, 2000.

6. Pandharipande P, Shintani A, Peterson J, Pun BT, Wilkinson GR, Dittus $\mathrm{RS}$, et al. Lorazepam is an independent risk factor for transitioning to delirium in intensive care unit patients. Anesthesiology. 2006;104(1):21-6.

7. Ely EW, Shintani A, Truman B, Speroff T, Gordon SM, Harrell Jr FE, et al. Delirium as a predictor of mortality in mechanically ventilated patients in the intensive care unit. JAMA. 2004;291(14):1753-62. https://dx.doi.org/10.1001/jama.291.14.1753

8. Kamdar BB, King LM, Collop NA, Sakamuri S, Colantuoni E, Neufeld KJ, et al. The effect of a quality improvement intervention on perceived sleep quality and cognition in a medical ICU. Crit Care Med. 2013;41(3):800-9.

https://dx.doi.org/10.1097/CCM.0b013e3182746442

9. Colombo R, Corona A, Praga F, Minari C, Giannotti C, Castelli A, et al. A reorientation strategy for reducing delirium in the critically ill. Results of an interventional study. Minerva Anestesiol. 2012;78(9):1026-33.

10. Needham DM, Korupolu R, Zanni JM, Pradhan P, Colantuoni E, Palmer JB, et al. Early physical medicine and rehabilitation for patients with acute respiratory failure: a quality improvement project. Arch Phys Med Rehabil. 2010;91(4):536-42. https://dx.doi.org/10.1016/j.apmr.2010.01.002

11. Reade MC, Finfer S. Sedation and delirium in the intensive care unit. N Engl J Med. 2014;370:444-54. https://dx.doi.org/10.1056/NEJMra1208705
12. Wolfe KS, Patel BK, Pohlman AS, Hall JS, Kress JP. Delirium and sedative requirements in a trial of helmet versus facemask noninvasive ventilation in acute hypoxic respiratory failure. Am J Respir Crit Care Med. 2017;195:A3012.

13. Centre Evidence-Based Medicine (CEBM). Oxford Centre for Evidence-based Medicine: Levels of Evidence (March 2009). Disponível em: http://www.cebm.net/oxford-centre-evidencebased-medicine-levels-evidence-march-2009/. Acesso em: 11 set. 2017.

14. Jeon $\mathrm{K}$, Jeong $\mathrm{BH}$, Ko MG, Nam J, Yoo H, Chung CR, et al. Impact of delirium on weaning from mechanical ventilation in medical patients. Respirology. 2016;21(2):313-20. https://dx.doi.org/10.1111/resp.12673

15. Lin SM, Huang CD, Liu CY, Lin HC, Wang CH, Huang PY, et al. Risk factors for the development of early-onset delirium and the subsequent clinical outcome in mechanically ventilated patients. J Crit Care. 2008;23(3):372-9.

https://dx.doi.org/10.1016/j.jcrc.2006.09.001

16. Lat I, McMillian W, Taylor S, Janzen JM, Papadopoulos S, Korth $\mathrm{L}$, et al. The impact of delirium on clinical outcomes in mechanically ventilated surgical and trauma patients. Crit Care Med. 2009;37(6):1898-905

https://dx.doi.org/10.1097/CCM.0b013e31819ffe38

17. Micek ST, Anand NJ, Laible BR, Shannon WD, Kollef $M H$. Delirium as detected by the CAM-ICU predicts restraint use among mechanically ventilated medical patients. Crit Care Med. 2005;33(6):1260-5.

https://dx.doi.org/10.1097/01.CCM.0000164540.58515.BF

18. Tsuruta R, Nakahara T, Miyauchi T, Kutsuna S, Ogino Y, Yamamoto $\mathrm{T}$, et al. Prevalence and associated factors for delirium in critically ill patients at a Japanese intensive care unit. Gen Hosp Psychiatry. 2010 32(6):607-11.

https://dx.doi.org/10.1016/j.genhosppsych.2010.09.001

19. Spronk PE, Riekerk B, Hofhuis J, Hommes JH. Occurrence of delirium is severely underestimated in the ICU during daily care. Intensive Care Med. 2009;35(7):1276-80. https://dx.doi.org/10.1007/s00134-009-1466-8

20. Mehta S, Cook D, Devlin JW, Skrobik Y, Meade M, Fergusson $D$, et al. Prevalence, risk factors, and outcomes of delirium in mechanically ventilated adults. Crit Care Med. 2015;43(3):557-66. https://dx.doi.org/10.1097/CCM.0000000000000727

21. McConville JF, Kress JP. Weaning patients from the ventilator. N Engl J Med. 2012;367(23):2233-9. https://dx.doi.org/10.1056/NEJMra1203367 
22. Dessap AM, Roche-Campo F, Launay JM, Charles-Nelson A, Katsahian S, Brun-Buisson C, et al. Delirium and circadian rhythm of melatonin during weaning from mechanical ventilation: an ancillary study of a weaning trial. Chest. 2015;148(5):1231-41. https://dx.doi.org/10.1378/chest.15-0525

23. Ansaloni L, Catena F, Chattat R, Fortuna D, Franceschi C, Mascitti P, et al. Risk factors and incidence of postoperative delirium in elderly patients after elective and emergency surgery. Br J Surg. 2010;97(2):273-80. https://dx.doi.org/10.1002/bjs.6843

24. Reade MC, Eastwood GM, Bellomo R, Bailey M, Bersten A, Cheung B, et al. Effect of Dexmedetomidine added to standard care on ventilator-free time in patients with agitated delirium: a randomized clinical trial. JAMA. 2016;315(14):1460-8. https://dx.doi.org/10.1001/jama.2016.2707

25. Arias-Rivera S, Sánchez-Sánchez Mdel M, Santos-Díaz R, Gallardo-Murillo J, Sánchez-Izquierdo R, Frutos-Vivar F, et al. Effect of a nursing-implemented sedation protocol on weaning outcome. Crit Care Med. 2008;36(7):2054-60. https://dx.doi.org/10.1097/CCM.0b013e31817bfd60

26. Kress JP, Pohlman AS, O'Connor MF, Hall JB. Daily interruption of sedative infusions in critically ill patients undergoing mechanical ventilation. N Engl J Med. 2000;342(20):1471-7. https://dx.doi.org/10.1056/NEJM200005183422002

27. Pisani MA, Murphy TE, Araujo KL, Slattum P, Van Ness PH, Inouye SK. Benzodiazepine and opioid use and the duration of intensive care unit delirium in an older population. Crit Care Med. 2009;37(1):177-83 https://dx.doi.org/10.1097/CCM.0b013e318192fcf9

28. Agarwal V, O'Neill PJ, Cotton BA, Pun BT, Haney S, Thompson J, et al. Prevalence and risk factors for development of delirium in burn intensive care unit patients. J Burn Care Res. 2010;31(5):706-715. https://dx.doi.org/10.1097/BCR.0b013e3181eebee9
29. Mehta S, Burry L, Cook D, Fergusson D, Steinberg M, Granton J, et al. Daily sedation interruption in mechanically ventilated critically ill patients cared for with a sedation protocol: a randomized controlled trial. JAMA. 2012;308(19):1985-92 https://dx.doi.org/10.1001/jama.2012.13872

30. Vasilevskis EE, Ely EW, Speroff T, Pun BT, Boehm L, Dittus RS Reducing latrogenic Risks: ICU-acquired delirium and weakness crossing the quality chasm. Chest. 2010;138(5):1224-33. https://dx.doi.org/10.1378/chest.10-0466

31. Leite MA, Osaku EF, Costa CR, Cândia MF, Toccolini B, Covatti $C$, et al. Delirium during weaning from mechanical ventilation. Crit Care Res Pract. 2014;2014:546349. https://dx.doi.org/10.1155/2014/546349

32. Ruokonen E, Parviainen I, Jakob SM, Nunes S, Kaukonen M Shepherd ST, et al. Dexmedetomidine versus propofol/midazolam for long-term sedation during mechanical ventilation. Intensive Care Med. 2009;35(2):282-90 https://dx.doi.org/10.1007/s00134-008-1296-0

33. Maldonado JR, Wysong A, van der Starre PJ, Block T, Miller C, Reitz BA. Dexmedetomidine and ther eduction of postoperative delirium after cardiac surgery. Psychosomatics. 2009;50(3):206-17. https://dx.doi.org/10.1176/appi.psy.50.3.206

34. Ho KM, Ng JY. The use of propofol for medium and long-term sedation in critically ill adult patients: A meta-analysis. Intensive Care Med. 2008;34(11):1969-79 https://dx.doi.org/10.1007/s00134-008-1186-5

35. Hopkins RO, Jackson JC. Assessing neurocognitive outcomes after critical illness: are delirium and long-term cognitive impairments related? Curr Opin Crit Care. 2006; 12(5):388-94 https://dx.doi.org/10.1097/01.ccx.0000244115.24000.f5 